\title{
A Preliminary Study on Evaluation Model of Bullacta exarata Invasion in
}

\section{the Yellow River Delta}

\author{
Tianwen ZHANG, Tong LIU、Yingjun WANG, Yan ZOU, YINGying WU, Aihuan
}

SONG**, Wen GUO

(Marine Biology Institute of Shandong Province, Shandong, Qingdao,266002;

Engineering laboratory for data mining and utilizing germplasm resources of marine organisms of Qingdao , Shandong ,Qingdao, 266002)

\section{Key Words: Bullacta exarata; Yellow River Delta; evaluation model}

Abstract: In recent years, with the rapid development of Chinese economy and the increase of the immigration, more and more alien species which cause a certain ecologic influence on the local area are coming in China. Risk assessment is the first step to prevent invasion of alien species, which is one the most effective measures. Ecological risk of alien species Bullacta exarata in Yellow River Delta was evaluated by this article. The model of risk assessment of Bullacta exarata was suggested. The invasion risk of Bullacta exarata evaluation index system was explored, hoping to provide reference for risk assessment of invasive alien species.

Commonly known as "wheat snail" and "say's paper-bubble", Bullacta exarata is the member of genus Bullacta exarata, family atyidae, order cephalaspide, class gastropoda and phylum Mollusca. As the species of eurythermal and euryhaline organism, Bullacta exarata is mainly reproduced in Ningbo and the Eastport of Liaoning. In 1980s, No Bullacta exarata was found in the investigation of Yellow River Delta. In 2001, some farmers in Kenlin County introduced seeds of Bullacta exarata from Jiangsu, Liaoning, etc. and also spread the seeds in intertidal zone of Yellow River Estuary by bottom sowing for culture and proliferation. In the second year, high economic revenue was obtained. The sea area of Yellow River Estuary is large, which is why the seeds can greatly increase by proliferation in distribution scope without planting. Until 2004, Bullacta exarata was found in the estuary area $50 \mathrm{~km}$ away from initial point of planting, while the Bullacta exarata became the advantageous species in some estuary areas of middle and low tide, having a maximum population density up to thousands snails per square meter ${ }^{[1]}$. As a foreign species, Bullacta exarata affects the local ecological environment and benthonic organisms after it became the advantageous species after mass propagation in natural waters beyond aquiculture area.

The rapid economic development, development of trade and traffic system as well as the increase in people entering and exiting help to promote the introduction of alien species ${ }^{[2]}$. The hazard and quarantine of foreign plant diseases and insect pests have drawn attention from Chinese departments of quarantine, customs, agriculture, forest and so on for a long time, but the ecological concept and significance in biological diversity protection of alien invasive species were just recently introduced to China ${ }^{[3]}$. Marine biotic intrusion, ocean pollution, overfishing of fishery resources and habitat destruction have become four major problems in the world marine ecology ${ }^{[4]}$.

\footnotetext{
** Corresponding author: Aihuan SONG : (1978-), Associate Researcher, Engaged in Marin Ecology。E-mail:zjusah@163.com。 Author: Tianwen ZHAGN (1983-), Doctor, Engaged in Model Specification。E-mail:ztw236@163.com。
} 
As a large marine province in China and especially in the Yellow Delta region, Shandong provides natural conditions for alien species with vast ocean space. Some invasive species can affect the local species by altering the utilization of environmental conditions and resources. It may not only reduce the biological diversity, but it may also affect the energy flow, material circulation as well as other functions of the system. If the influence is severe, the whole ecological system may collapse [5]. Foreign marine species may threaten local species and make these species extinct by competing with the local species. Therefore, the species diversity of community is affected, reducing the biological diversity and changing the constitution of community.

\section{Changes of Bullacta exarata Resources in "Yellow River Delta"}

Located in the seacoast of Bohai Sea, Yellow River Delta has long coastline, vast mudflat as well as rich organic substances in seawater and sufficient bait. Its shellfish resource is rich, mainly including rubromuscula, lusoria, Mactra veneriformis and cyclina sinensis in areas that are more than 5.6 million mu with yield of more than 300 thousand tons. Therefore, this is an important area of shellfish resource ${ }^{[6]}$. According to historical investigation, there was no Bullacta exarata in Yellow River Delta in 1980s . Because of its introduction, a large amount of Bullacta exaratas entered the Yellow River Delta. In 2005, the beach face near Weifang Port in the south of Laizhou Bay also became the distribution area of Bullacta exarata. Presently, Bullacta exarata has become the dominant species in the intertidal zone of this coastal segment. In 2007, the occurrence frequency of Bullacta exarata in the middle and low tidal areas at the west bank of Laizhou Bay was about $80 \%$. In 2008, the distribution area of Bullacta exarata in the southern bank of the Bohai Sea further expanded and Bullacta exarata became the dominant species in beach face and most coastal segments in the southern bank of Bohai Bay, as well as intertidal zone in the western bank of Laizhou Bay. As shown in Fig. 1, Bullacta exarata has mass propagation in Yellow River Delta as an alien species. Until 2013, the density of Bullacta exarata has been about $23.24 \mathrm{ind} / \mathrm{m}^{2}$.

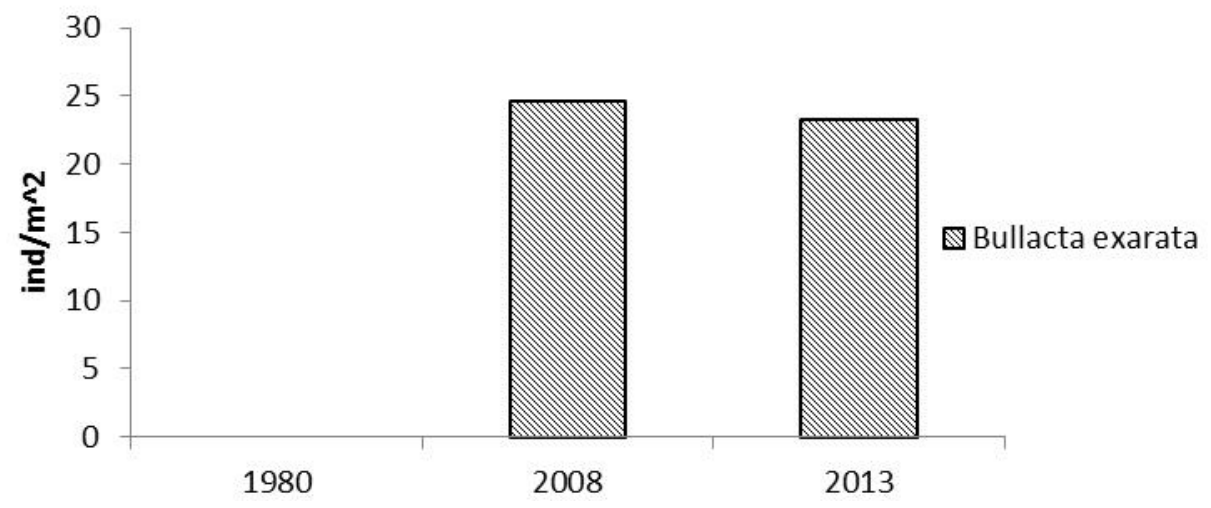

Fig.1 Density of Bullacta exarata varies with time

\section{Introduction of Intrusion Risk Evaluation Model for Alien Species}

Yellow River Delta ecological system is a complex ecological system, which is composed of dynamic and stereo marine ecological system, land system closely related to ocean and socio-economic system of human. A complex interaction among these units exists. All systems and their units have dynamic characteristics of self-control, successive capabilities, etc. The complexity of ecological system in Yellow River Delta determines the complexity of its safety evaluation system as well as its ecological safety prediction. PSR model is selected as an evaluation model when evaluating the intrusion risks of Bullacta exarata in Yellow River Delta. 
PSR model (Pressure-State-Response model) was firstly proposed by Canadian statisticians, Tony Friend and David Rapport for analyzing the relationship between environmental pressure, state and response ${ }^{[7]}$. In PSR model, the logical thinking of pressure-state-response is used when selecting indicators. The objective of model construction is to provide answers to what happens, why it happens and how to respond to it ${ }^{[8]}$. Based on causal relationship, PSR model reflects the interaction between human and environment, in other words, human activities apply a certain pressure on natural resources. Because of the external pressure, natural resources change the original state and humans will respond to these changes by altering environmental, economic and management strategies. Therefore, natural resources can be recovered and environmental degradation can be prevented. Furthermore, pressure variable is used to describe the influences of human activities on the environment, state variable is used to describe the physical measurable characteristics of environmental issues caused by pressure variable and respond variable is used to describe the degree that the society responds to environmental issues. Nonetheless, the respond variable can directly or indirectly affect the changes of state variables. Such circulation constitutes the pressure-state-respond reslationship between humans and environmental resources ${ }^{[9]}$.

In PSR model for intrusion risk evaluation of Bullacta exarata, the subjects of marine development, producers and managers were unaware of the pressure of introduction of Bullacta exarata on marine environment and fishery resources at the beginning. With the ongoing development, pressure increases gradually and it is reflected through the reduction of fishery resources. The release of such pressure may affect social subjects who, then, react to decision-making. Fig. 2 shows the PSR model for intrusion risks of Bullacta exarata. In this evaluation system, $\mathrm{P}$ represents the pressure of Bullacta exarata intrusion to fishery resources, $\mathrm{S}$ represents the quality and state of fishery resource, $\mathrm{R}$ represents the reaction of managers and producers to such influences ${ }^{[10]}$.

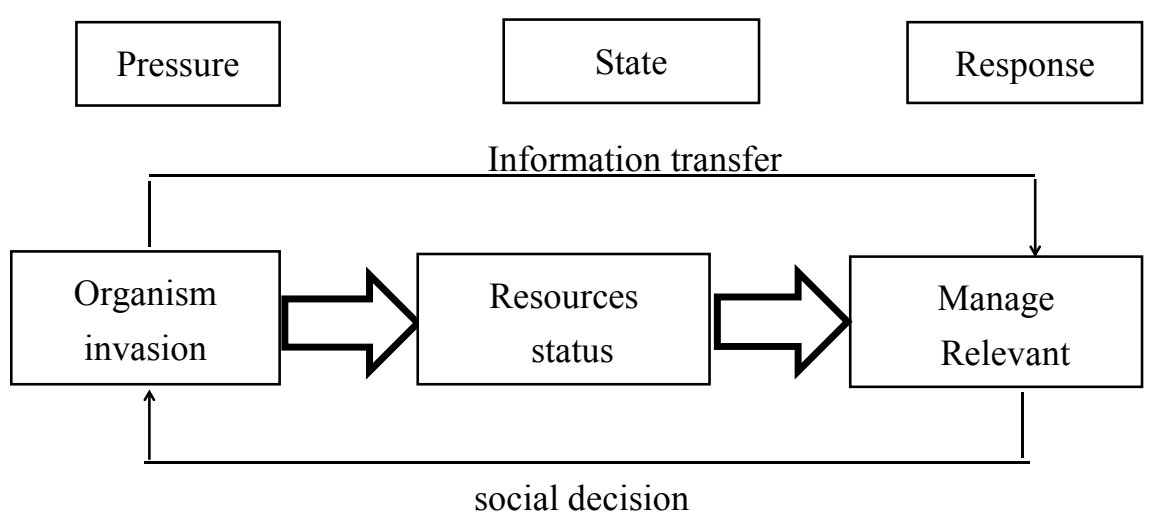

Fig.2 Evaluation system of organism invasion

\section{Technical Route and Data Processing of Evaluation System}

According to collected historical resources and data from supplementary investigation, intrusion risk evaluation system is screened for Bullacta exarata with regional scope, yield, individual density and other biological parameters and fishery resource species. The ratio of fishery production in a specific sea area is used as basic indicator in the principle of science, application and integrity. Analytic hierarchy process is used to determine the weight of evaluation indicator and multi-level fuzz comprehensive evaluation can be used to construct the intrusion risk evaluation for Bullacta exarata.

Fig. 3 shows the main technical route of evaluation. The intrusion risk of Bullacta exarata is analyzed according to the changes in the yield of main fishery resources as well as the 
environmental changes in sea area. Evaluation indicators are thereby screened for intrusion risks of Bullacta exarata in order to establish the evaluation system for intrusion risk of Bullacta exarata.

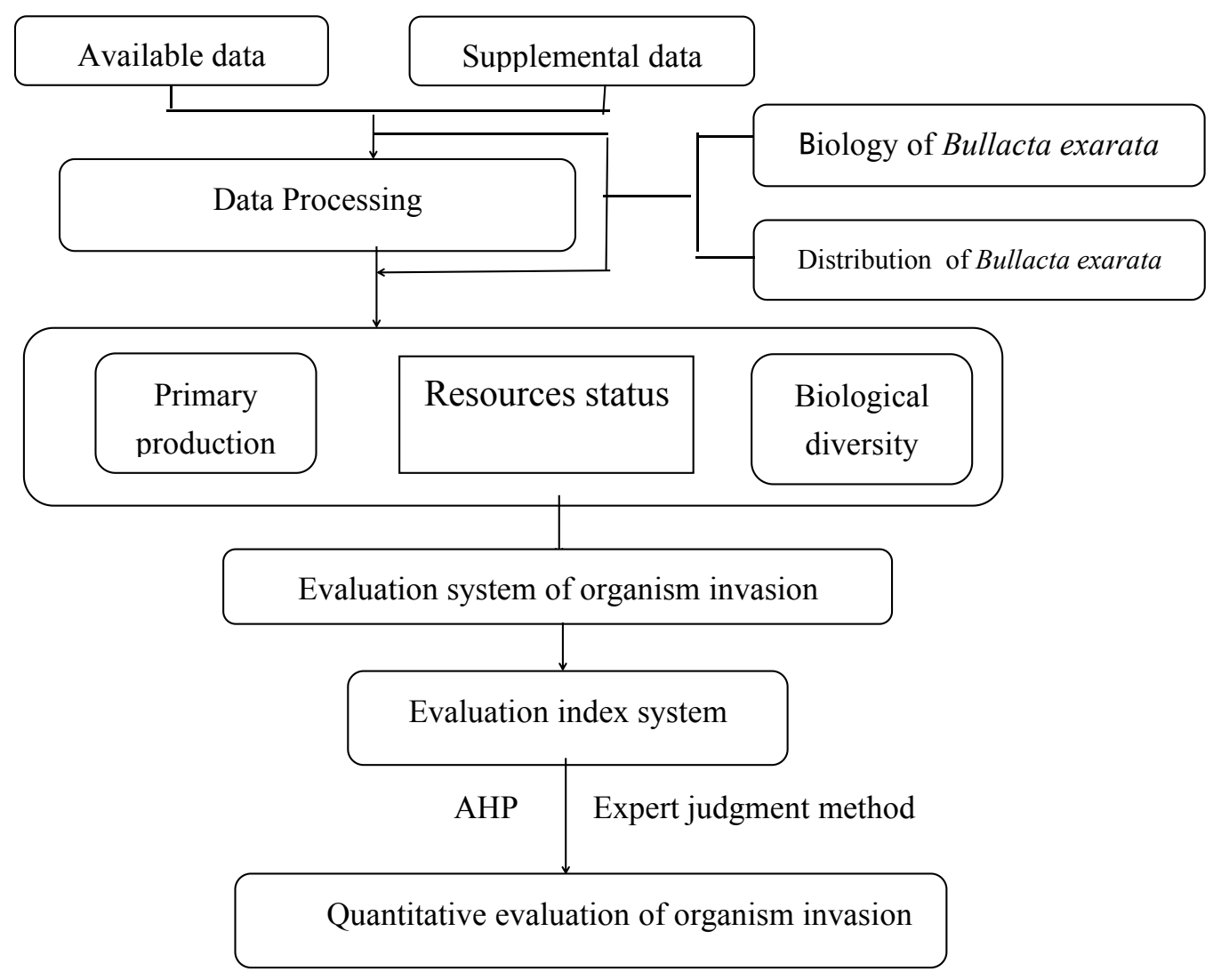

Fig.3 The technological route for organism invasion

\section{Weight Determination and Data-Reliability Analysis for Indicator System}

To determine the degree of intrusion risks of Bullacta exarata, the evaluation system should be established and the weight of all indicators should be determined [11-16]. Presently, the weight of evaluation indicator is determined by two methods at home and abroad.

The first method is subjective weighting, including analytic hierarchy process and Delphi method, which are qualitative methods for weight determination. Generally, the weight will be obtained by subjective judgment according to experience and indicators will be comprehensively evaluated. Zhao Guihong et al. combined expert consultation with the actual situation of safety management in airport by Delphi method so as to determine the weight of safety indicators in parking apron. Guo Yanying et al. established a scientific comprehensive evaluation system for surface water quality and also determined the weights of indicators for comprehensive evaluation of surface water quality.

Another method is objective weighting, including entropy evaluation method and principal component analysis, which are dependent on the actual problem without subjectivity and arbitrariness. Without increasing the burden of decision maker, weight is determined by a mathematic method according to the relation between original data, so the judgment is greatly based on mathematical theories ${ }^{[17]}$. Bao Lei et al. determined the main indicators that affect the safety in metro system by principal component analysis and also ranked the safety degree of 4 sections in the metro system.

There is no sufficient sample data and actual problem field when determining the evaluation weight of Bullacta exarata invasion risks, it is better to use subjective scoring for analysis. In 
addition, there are various indexes for evaluating the Bullacta exarata invasion risks with massive data, it is highly difficutl to reach consistency by analytic hierarchy process. On the contrary, by delphi method, multiple rounds of expert scoring are required to ensure the sufficient reflection of expert opinions and reliability of final conclusions with multiple feedbacks. Therefore, delphi method is used to evaluate Bullacta exarata invasion risks ${ }^{[18-19]}$.

\section{Construction of Evaluation System for Intrusion Risk of Bullacta exarata}

The evaluation system for intrusion risk of Bullacta exarata is established under the influence of Bullacta exarata intrusion on local fishery resources, having economic and social development as its overall objective. Based on the influential factors, the system is analyzed with full consideration into evaluation indicators. The basic framework of this model comprises of target layer, criteria layer and indicator layer. Target layer is used to determine the influence degree of Bullacta exarata intrusion on local fishery economy. Criteria layer is the intermediate link of evaluation indicator reaching the preset target. Indicator layer is the characteristic indicator which has typical representative significance and effectively reflects the intrusion risk of Bullacta exarata.

Intrusion risk and effect evaluation system of Bullacta exarata is constructed based on the analytic framework of PSR model for intrusion risk evaluation of Bullacta exarata in principle of selecting typical, systematic and quantitative indicators. The indicator system is divided into target layer, criteria layer and indicator layer, including 12 specific indicators.

Table.1 Intrusion risk and effect evaluation system of Bullacta exarata

\begin{tabular}{|c|c|c|c|}
\hline Target Layer & Criteria Layer & \multicolumn{2}{|c|}{ Indicator Layer } \\
\hline \multirow{6}{*}{$\begin{array}{l}\text { Intrusion risk and effect } \\
\text { evaluation system of } \\
\text { Bullacta exarata }\end{array}$} & \multirow{3}{*}{ Pressure } & $\begin{array}{c}\text { The invasion of Bullacta } \\
\text { exarata }\end{array}$ & $\begin{array}{c}\text { Introduction of Bullacta } \\
\text { exarata }\end{array}$ \\
\hline & & \multirow[t]{2}{*}{ Status } & $\begin{array}{c}\text { Biomass of Bullacta } \\
\text { exarata }\end{array}$ \\
\hline & & & Density of Bullacta exarata \\
\hline & \multirow[b]{2}{*}{ State } & Ecological condition & $\begin{array}{l}\text { Primary productivity } \\
\text { Diversity of benthos }\end{array}$ \\
\hline & & $\begin{array}{l}\text { Benthic fishery } \\
\text { resources }\end{array}$ & $\begin{array}{c}\text { Lusoria resource } \\
\text { Mactra veneriformis } \\
\text { Cyclina sinensis } \\
\text { Rubromuscula } \\
\text { Tellinidae resource }\end{array}$ \\
\hline & Response & $\begin{array}{l}\text { Environmental } \\
\text { protection }\end{array}$ & $\begin{array}{c}\text { Manual elimination of } \\
\text { Bullacta exarata } \\
\text { Enhancement and releasing } \\
\text { native species }\end{array}$ \\
\hline
\end{tabular}

\section{Acknowledgements}

This work was financially supported by Key research and development projects of Shandong province (2016GSF115007), Evaluation and utilization of database establishment for aquaculture germplasm resources ,China CNOOC Charity Foundation Project (2016-2020), Major projects of China association of marine affairs and The earmarked fund for Modern Agro-industry Technology 
Research System（SDAIT-14）.

\section{References}

[1]OCCHIPINTI-AMBROGI A. GALILBS.A uniform terminology on bioinvasions : A chimera or an operative tool[J] .Marine Pollution Bulletin,2004,49:688 - 694.

[2] XU Hai-Gen, QIANG Sheng, HAN Zheng-Min, The distribution and introduction pathway of alien invasive species in China[J]. Biodiversity Science, 2004,12(6):62663.8

[3] MA Chenglin1, ZOU Jixing. MARINE BIODIVERSITY AND ITS CONSERVATION IN CHINA SEAS[J]. Transactions of Oceanology and Limnology. 2003,(2):41-47.

[4] YANG Sheng-yun, WU Li-sheng,CHEN Ming-ru. Exotic species of marine propagation and marine ecology conservation[J]. JOURNAL OF OCEANOGRAPHY IN TAIWAN STRAIT.

2001,20(2):259-265.

[5]ZHAO Zhang-yuan, Kong Ling-hui. Environmental Status Quo and Protection Countermeasures in Bohai Mar ine Areas[J]. Research of Environmental Sciences, 2000,13 (2): 23-27.

[6] LIU Hong-wei,et al. Sustainable Utilization of Marine Fishery Resources of Bohai Sea[J]. Journal of Anhui Agri.Sci, 2010,38 (26): 14579-14581.

[7]P. Baake, and A. Boom, Vertical Product Differentiation, Network Externalities, and Compatibility Decisions[J].International Journal of Industrial Organization 2001， 19: 267-284.

[8]Guo Yanying,Deng Yunfeng,Ren Jun. Application of Analytic Hierarchy Process(AHP)Method in Comprehensive Assessment of Surface Water Quality [J]. Journal of Lanzhou Jiaotong University, 2006, 25 (3): 70-76.

[9] ZHAO Gui-hong, TIAN Sha-sha. Study on Safety Factors Choice for Airport Apron Based on Delphi Method[J]. JOURNAL OF CIVIL AVIATION UNIVERSITY OF CHINA.2008, 26（6):

61-64.

[10] LIXian-sen,NIUMing-xiang,DAIFang-qun. The Characteristics of Structure and distribution of spawning stock of fishery species in the Bohai Sea[J]. MARINE FISHERIES RESEARCH , 2008,29(4):15-21.

[11]THRESHER R E, KURIS A M .Options for managing invasive marine species [J]. Biological Invasion,2004,6:295-300.

[12] Xiang Yanci, Peng Shaolin, Ren Hai. Management and Ecological Risk Assessment of Exotic Plants. [J]. Chinese Journal of Ecology.2002,21(5):40-48

[13] MA yan,ZHENG xiang-min. STUDY ON ECOLOGICAL RISK ASSESSMENT[J]. TERRITORY \& NATURAL RESOURCES STUDY,2005,(2):49-51.

[14] ZHU Lin, TONG Yu-jie. CASE RESEARCH AND ISSUE DISCUSSION OF ECOLOGICAL RISK ASSESSMENT[J]. Journal of Safety and Environment.2003,3(3):22-24.

[15] MAIShao-zhi,XU Song-jun,PAN Ying-jun. APPLICATION OF THE PSR MODEL TO THE EVALUATION OF WETLAND ECO SYSTEM HEALTH[J]. TROPICAL GEOGRAPHY, 2005, 25 (4): 317-321.

[16] YU Ding-Yong,WANG Chang-Hai,LIU Hong-Chao. Study on Reclamation Impact on Marine Resources Based on PSR Model[J]. PERIODICAL OF OCEAN UNIVERSITY OF CHINA, 2011,41(7/8): 170-175.

[17] YANG Zhi,ZHAO Dong-zhi,LIN Yuan-shao. The evaluating indexsystem for estuary ecosystem safety based on PSR model[J]. MARINE ENVIRONMENTAL SCIENCE , 2011,30(1):138-141.

[18] SHEN Yong-ming, LIUYong-mei,CHENQuan-zhan. Analysis of the expanding process of the Spartina alterniflora Loisel salt marsh on Jiangsu Province coast by remote sensing[J]. Journal ofPlant Resources and Environment.2002,11(2):33-38.

[19] CHENZhong-Yi,LIBo,CHENJia-Kuan. 'Ecological consequenees and management of Spartina spp.invasions in coastal ecosystems[J]. Biodiversity Science,2004,12(2):280-289. 